\title{
Design and Implementation of Optimum LSD Coded Signal Processing Algorithm in the Multiple-Antenna System for the 5G Wireless Technology
}

\author{
Priyanka Pateriya $\mathbb{D}^{1},{ }^{1}$ Rakesh Singhai, ${ }^{2}$ and Piyush Shukla ${ }^{3}$ \\ ${ }^{1}$ Rajiv Gandhi Technical University, Bhopal, India \\ ${ }^{2}$ University Institute of Technology RGPV, Shivpuri, India \\ ${ }^{3}$ UIT-Rajiv Gandhi Technical University, Bhopal, India
}

Correspondence should be addressed to Priyanka Pateriya; priyankapateriya03@gmail.com

Received 11 January 2022; Revised 23 January 2022; Accepted 27 January 2022; Published 25 February 2022

Academic Editor: Mohammad Farukh Hashmi

Copyright (c) 2022 Priyanka Pateriya et al. This is an open access article distributed under the Creative Commons Attribution License, which permits unrestricted use, distribution, and reproduction in any medium, provided the original work is properly cited.

\begin{abstract}
The 5G system requires an optimum coding technique to achieve the high diversity gain, low bit error rate (BER), and low detection complexity. Various coding techniques were developed in recent times for improving the diversity performance of the MIMO systems. Space-time-coding (STC) is used to fulfill the requirement of handling large data flow in the 5G wireless communications. It is highly required to optimize the orthogonal nature of STC. The paper proposed a novel design of the optimum linearly scalable dispersion code (O-LSDC). In this paper, an optimum coefficient-based O-LSDC is designed based on the elementary matrix operations, unitary matrix normalization technique, and coefficient mapping strategy. Mapped coefficients are linearly solved for optimum value estimation. To find the optimum solution of the LSDC codes, five cases of LSDC are defined based on the scaling coefficients and then performance is evaluated against the BER vs. SNR. Evaluating the simulation results in terms of error probabilities for the five different orthonormal LSDC, this work simulates the system for multiple antennas using the Rayleigh fading MIMO system model. Also, evaluating the impact of the proposed LSDC over the BER performance for the varied number of Monte Carlo iterations, then the performance graph is plotted for multiple-antennas system. The proposed O-LSDC under Rayleigh fading channel using the M-PSK modulation enhances the performance of the 5G and beyond communication system in terms of BER and SNR.
\end{abstract}

\section{Introduction}

Nowadays, there is a significant information overload on the channels of wireless communication. Especially during the COVID-19 pandemic, the digital information flow is significantly increased, which demands enhancing the wireless channel capacity. The 5G wireless system with the massive MIMO technique is used to improve the capacity of the wireless channel. In the multiple-antenna system, the channel between transmitter and receiver is modeled with multiple paths. This offers the wireless channels to be modeled as the Rayleigh fading channel. Practicable modulation technique for multiple-antenna systems with receive- $(\mathrm{Rx}-)$ only channel information comes under two categories termed as diversity and multiplexing. Due to different gains and phases, the MIMO offers diversity in the space domain, popularly known as spatial diversity to maximize the reliability of the transmitted data. Spatial multiplexing performs the simultaneous/parallel transmission of the individual data streams from every transmitting (Tx) antenna to enhance the channel capacity at the cost of diversity in the fading channels. The practicable systems required new space-time (ST) signaling schemes to achieve high reliability (diversity) and high spectrum efficiency (SE) $[1,2]$.

While using OSTBC reduces channel capacity in MIMO channels while increasing diversity, a revolutionary ST coding known as linear scalable dispersion codes is 
suggested (LSDC). LSDC utilizes a linear matrix modulation scheme, in which the transmitted codeword is a linear combination of the dispersion matrices with the weights evaluated by the Tx symbols; the coefficients of these matrices are selected in such a way that it increases the ergodic capacity of the multiple-antenna system $[3,4]$. This code is a special case of the STBC $[5,6]$.

The MIMO system using LSDC and MMSE receiver in a Rician flat-fading environment is studied in $[7,8]$. The encoded data is Tx towards the eigenvectors of the squared Rician channel components and it is found out that the optimum power allocates approach and determined the bounds on Pe for various Tx criteria. This work proved that the best Tx strategy for low SNR is the beamforming approach [9-11]. Implementation of space frequency- (SF-) LSDC is studied in $[10,12,13]$, which requires an appropriate linear precoding method and derived ST dispersion matrix to achieve full Tx diversity-multiplexing advantages. For the frequency selective channel, SF-LDC is more appropriate in contrast to ST-LSDC, but both induce high PARP in this system [14]. The U-LSDC is proposed in [10] by converting the $M \times M$ LSDC into $T \times M$ nonsquare LSDC, where $M$ is the code rate. U-LSDC illustrates the unitary encoding rectangular matrix $[15,16]$. It is mathematically traceable and provides symbolwise full diversity. The LDC designed from genetic algorithms aided optimization approaches provides average pairwise error probability (PEP), high data rate, and optimized $\mathrm{MI}$ at the cost of detection complexness [14-23].

Up till now, the LDCs are designed to increase the MI amid Tx and Rx end but do not care about the error probability $\left(P_{\mathrm{e}}\right)$ performance. Utilizing elementary matrix operations, unitary matrix normalization technique, and coefficient mapping strategy, as well as Monte Carlo simulation, this work proposed a novel design of the optimum linearly scalable dispersion code (O-LSDC) that maximizes the ergodic capacity and performs well in terms of BER and SNR, which was also found to be very efficient for any configuration of Tx and Rx antennas and codeword length. This paper demonstrates the LSDC design depends on the coefficient mapping strategy. Mapped coefficients are linearly solved to estimate the optimum coefficient values. Based on scaling coefficients, the paper presents the five versions of the LSDC for evaluation. The primary contribution of this paper is to design the optimum LSD coding method that enhances the performance of the multiple-antenna communication system under Rayleigh fading channel for the 5G networks in terms of error probabilities and maximum achieved signal for reliable data transmission. Outlining the offerings of this paper, we have the following:

(i) Describing the Validation of STBC-MIMO for the effective provisioning of the communications networks.

(ii) Proposing the system model to improve the efficiency of existing LSDC code based on channel realization. It is proposed to design the optimum space code vector for the orthonormal matrix for a communications system modeled with multipleantenna channels.

(iii) Presenting the five different cases of the LSDC.

(iv) Evaluating the simulation results in terms of error probabilities and comparing the BER vs. SNR for the various proposed LSDC codes for different rounds of Monte Carlo simulation for the NRD distribution and the performance graph are plotted sequentially for multiple-antenna systems, eventually, evaluating the results of the BER for the MIMO-O-LSDC system with the different higher-order PSK modulation techniques.

(v) Finally, presenting the conclusion of this work.

\section{STBC Coding}

The LSDC is a special case of the STBC implemented in orthogonal space used to achieve diversity goal. The orthogonal area-based OSTBC codes achieve good diversity in the space and time domains. These STBC codes can be represented as orthonormal matrix form of dimension $\mathrm{n}_{\mathrm{s}} \mathrm{x} \mathrm{N}_{\mathrm{t}}$, where $n_{\mathrm{s}}$ is the number of time instances and $N_{\mathrm{t}}$ is the number of Tx antennas expressed as follows [1-3]:

$$
\left[\begin{array}{cccc}
s_{11} & s_{12} & \ldots & s_{1 N_{t}} \\
s_{21} & s_{22} & \ldots & s_{2 N_{t}} \\
\vdots & \vdots & \ddots & \vdots \\
s_{\mathrm{n}_{s} 1} & s_{\mathrm{n}_{s} 2} & \ldots & s_{\mathrm{n}_{\mathrm{s} N_{t}}}
\end{array}\right] .
$$

The efficiency of the MIMO system depends on the efficient design and representation of the $s_{i}^{m}$ matrix. Let the information symbols be given as

$$
s_{i}^{m}=\varnothing_{1}, \varnothing_{2}, \ldots \ldots \varnothing_{m} .
$$

Let the input vector $x_{i}^{s_{i}^{m}}$ represent the symbol as

$$
x_{i}^{s_{i}^{m}}=X_{1}, X_{2}, \ldots \ldots . . X_{m} .
$$

If variable $H$ is the MIMO channel realization matrix, with the classification of the various block-based coding methods under the presence of noise $\mathrm{N}$, the received and transmitting vectors $Y_{i}^{s_{i}^{m}}$ are defined as

$$
Y_{i}^{s_{i}^{m}}=H_{t} x_{i}^{s_{i}^{m}}+N .
$$

Equation (4) can be extended as the

$$
Y_{i}^{s_{i}^{m}}=H_{1} x_{i}^{s_{i}^{m}}+H_{2} x_{i}^{*}+N
$$

where $H_{1}$ and $\mathrm{H}_{2}$ denote effective channel matrices comprised of a concatenation of $\mathrm{H}_{\mathrm{t}}$ and the ${ }^{*}$ symbol represents the complex conjugate. In this work, it is considered to transmit only one ST block during every $n_{s}$ channel operation. The value of $n_{s}$ is set to 4 in the current work. And the numbers of transmitting antennas corresponding to the code vectors are set to $N_{t}=3$. Thus, the corresponding STBC vector for the size of the $\mathrm{N}_{\mathrm{t}} \mathrm{n}_{\mathrm{s}}$ for transmitted signals is defined as 


$$
S=G_{1} x_{i}^{s_{i}^{m}}+G_{2} x_{i}^{*},
$$

where $G_{1}$ and $G_{2}$ are complex ST mapping matrices. The corresponding space-time code vector STBC is designed as

$$
S_{j}^{i}=\sum_{i=1}^{N t} \sum_{j=1}^{N r}\left|Y_{i}^{s_{i}^{m}}-\sum_{i=1}^{N t} H x_{i}^{s_{i}^{m}}\right| .
$$

2.1. Validation of STBC-MIMO. In this paper $n$ optimum $\mathrm{O}$-LSDC code is introduced as a paradigm for improving the communication performance over Rayleigh fading channels using the MIMO system. Initially the validation of the existing Alamouti STBC based coding methodology is presented. The basic STBC code offers orthonormal complex code matrix $\mathrm{O}$ of size $n_{s} * N_{t}$. The validation of STBC is done for $4 \times 3$ complex orthogonal matrix, respectively, to $3 / 4$ rate system. For the validation of STBC-MIMO, the complex orthogonal matrix $(\mathrm{O})$ is considered as follows [10]:

$$
O=\begin{array}{lll}
1 & -2 & -3 \\
2+j & 1+j & 0 \\
3+j & 0 & 2 \\
0 & -3+j & 2+j
\end{array} .
$$

The expected value of BER is calculated as

$$
\text { ber }=\frac{1}{2} \operatorname{erfc}\left(\sqrt{\left(E / N_{0}\right)}\right),
$$

where $E_{0}$ represents the energy of the modulated signal and $N_{0}$ represents the normalized noise power density level. The ratio $\left(E / N_{0}\right)$ corresponds to the SNR of the system. The performance validates the symbol error rates (SER) and BER for the STBC-MIMO system as depicted in Figure 1, which depicts that it is highly desired to decrease the BER of the MIMO systems validated with STBC coding. As the validated BER of the order of $10^{-2}$ an $d 10^{-3}$ are achieved for QPSK and BPSK modulation with the matrix given in equation (8). The BER plotted in the figure is corresponding to the 1000 iterations of Monte Carlo simulations.

The BER is also validated for the orthonormal matrix of equation (8) for basic STBC in Figure 2. The performance is plotted and validated for the BPSK and the QPSK modulations, respectively. The MIMO system is simulated using the 3 transmitting antennas and the Alamouti STBC coding. The constellation values of the M-PSK modulations are modeled using the orthonormal code vectors $\mathrm{S}$ shown in equation (8).

\section{Proposed System Model}

In this paper, it is proposed to design the optimum space code vector for the orthonormal matrix for a communications system modeled with multiple-antenna channels. It is proposed to improve the efficiency of the existing LSDC code-based realization of the channel. The proposed channel model considers the Rayleigh fading channel. The performance is proposed to evaluate the M-PSK modulation by

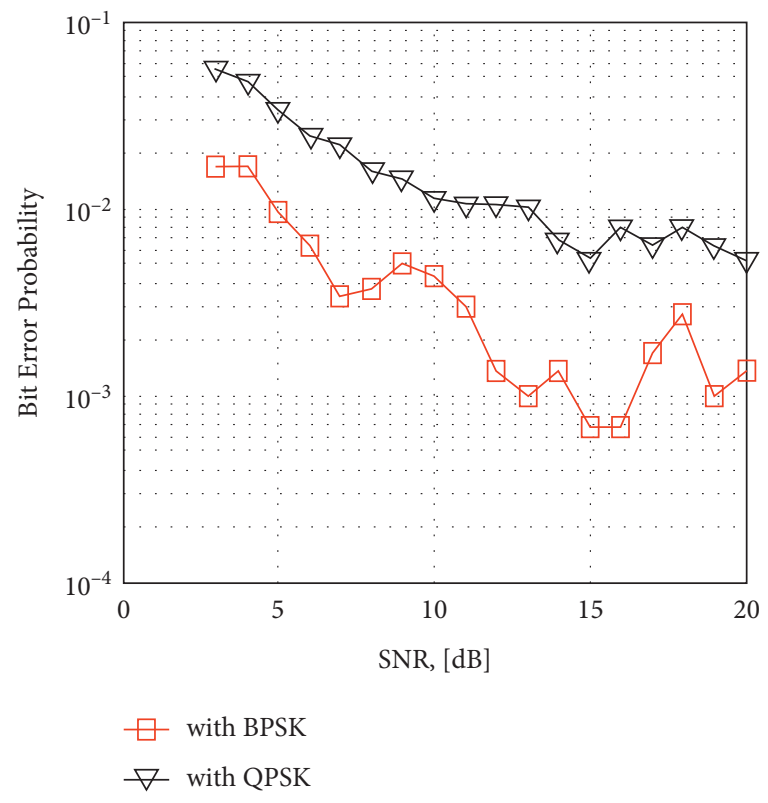

FIGURE 1: Validation of the BER performance for the BPSK and QPSK modulation using the existing STBC coding [9].

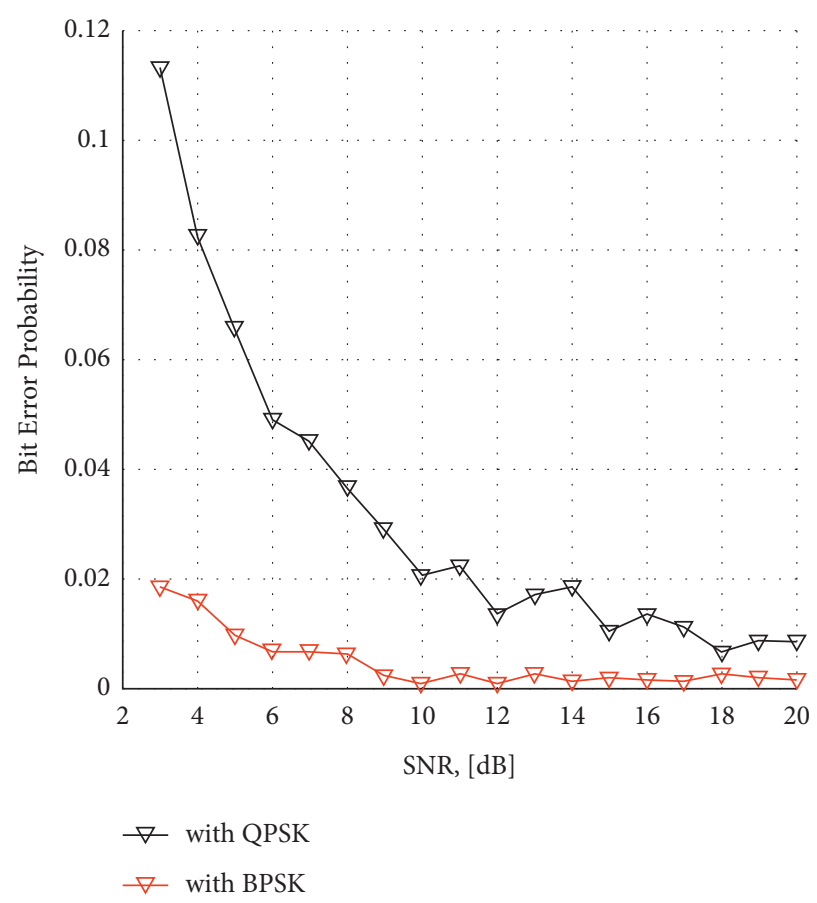

FIGURE 2: Validation of the basic STBC with the orthonormal matrix.

generating a random matrix of symbols. The LSD coded data is partitioned into $n$ data streams to be simultaneously transmitted utilizing $n \mathrm{Tx}$ antennas. The received signal at each $\mathrm{Rx}$ antenna is a linear superposition of the $n$ transmitted signals plus noise. The LSD coded signal is modeled using the Rayleigh channel and it is proposed to model the channel using the normalized channel realization matrix to improve the error rates of the system. The proposed method 
uses the NRD for the modeling system. The linear decision equalizer is used for the received signal processing with linear filtration method. It is also used for Tx data recovery and to mitigate the intersymbol interference. The received data is then decoded using inverse matrix RR scheduling using O-LSDC decoder and then demodulated using M-PSK to achieve the original data and to analyze the BER performance of the received data. The block diagram of the proposed system design is shown in Figure 3.where $s_{j}=\alpha_{j}+i \beta_{j}$, for $j=1, \ldots, 4$ are the transmitted symbols as a function of time taken from the QAM constellation. It is clear from Figure 4 that the performance of the system depends on the efficient channel realization and also using the efficient precoding design that can add the improvement. The LSD-based optimum precoding is proposed for design in this paper. The LSDC is a special coding method that is expected to improve the diversity of modeled multipleantenna channels. An example of LSDC for three antennas was acquired as [1].

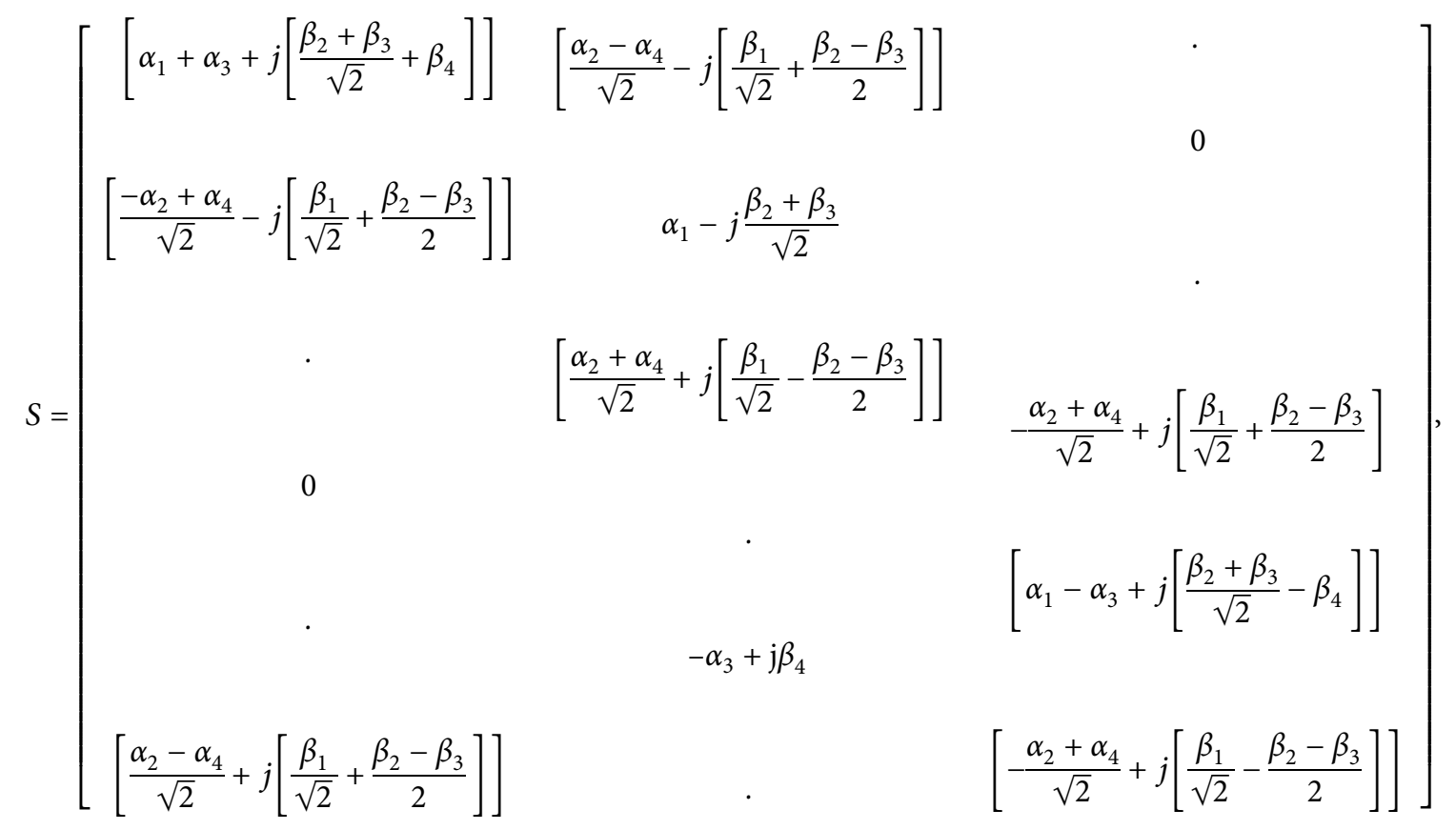

The research problem in this paper is to design the optimal $S$ matrix by experimentally deriving the optimal values of coefficients $\alpha_{1}$ to $\alpha_{4}$ and $\beta_{1}$ to $\beta_{4}$.

\section{Five Different Cases of LSDC}

In this work in favor to search for the optimum LSDC, five cases of LSDC are defined and then performance is evaluated against the SNR in $\mathrm{dB}$. The basic LSDC is defined in equation (10) by the orthonormal matrix. To derive the optimal coefficients values of $\alpha_{1}$ to $\alpha_{4}$ and $\beta_{1}$ to $\beta_{4}$, the real and imaginary terms of the $S_{i, j}$ term in equation (10) are compared, respectively, with equation (8).

\section{Case 1. LSDC-1}

As in Case 1 it was initially observed that most of the $\beta$ coefficients are having the multiple of $1 / \sqrt{2}$. Thus, as an experiment, it is tried to minimize the coefficients having value 0 in terms of $\sqrt{2}$ factors. The basic LSDC is defined in equation (10), which is the orthonormal matrix. In favor of deriving the optimal coefficients values of $\alpha_{1}$ to $\alpha_{4}$ and $\beta_{1}$ to $\beta_{4}$, the real and imaginary terms of the $S_{i, j}$ term in equation (10) are compared, respectively, with equation (8). Based on the relative comparison, the following linear equation combinations have been derived for finding the coefficients. Let the initial estimate be taken as

$$
\begin{aligned}
-\alpha_{3}+j \beta_{4} & =-3+j \longrightarrow \alpha_{3}=3 \text { and } \beta_{4}=1, \\
\frac{\beta_{2}-\beta_{3}}{2} & =0 \longrightarrow \beta_{2}=\beta_{3}=1 / \sqrt{2},
\end{aligned}
$$

set the $\alpha_{1}=1$, and try to set the $\alpha$ coefficients to eliminate the $\sqrt{2}$ factor as

$$
\begin{aligned}
\alpha_{2}+\alpha_{4} & =6 \sqrt{2} \longrightarrow \alpha_{2}=2 \sqrt{2}, \\
\alpha_{4} & =4 \sqrt{2}
\end{aligned}
$$

These linear equations lead to coefficient vectors as $\alpha_{1}=1, \alpha_{2}=2 \sqrt{2}, \alpha_{3}=3, \alpha_{4}=4 \sqrt{2}$, and 


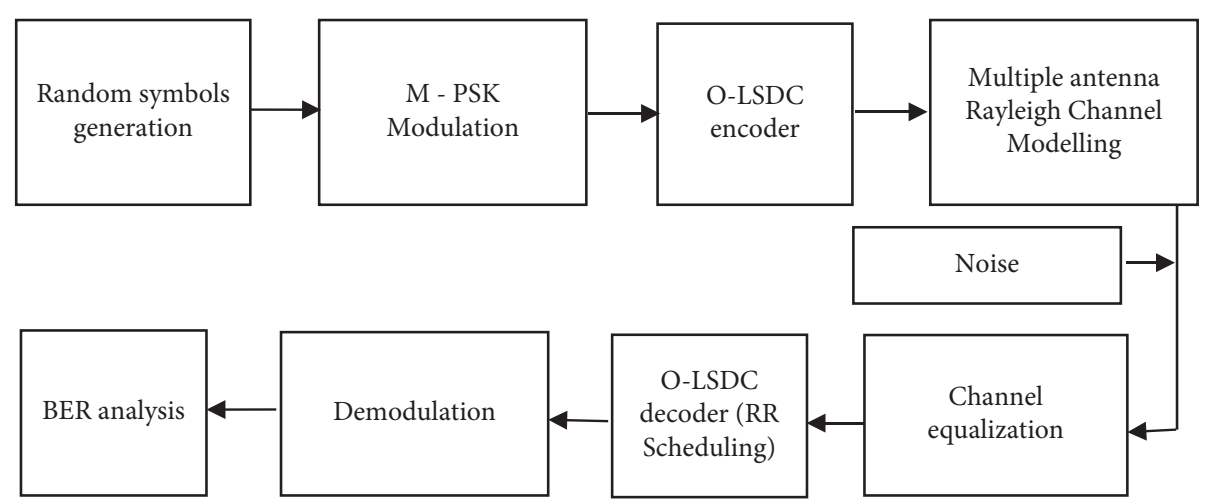

FIGURE 3: Block diagram of the proposed methodology.

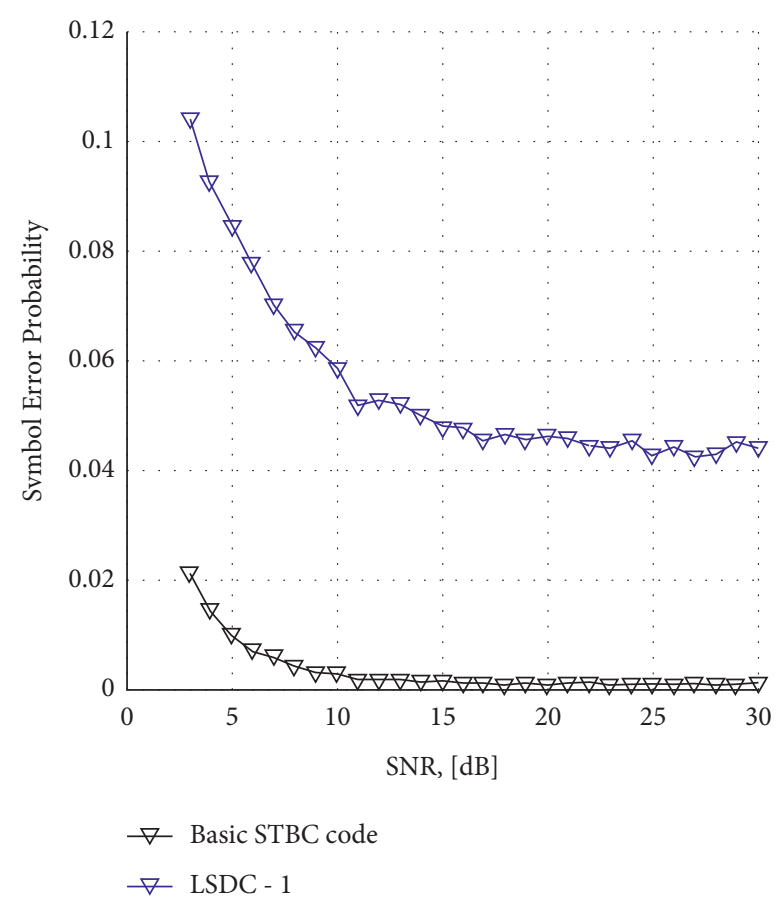

FIGURE 4: Comparison of SER for the LSDC-1 and the basic STBC performance.

$$
\beta_{1}=\sqrt{2} \beta_{2}=1 / \sqrt{2} \cdot \beta_{3}=1 / \sqrt{2} \cdot \beta_{4}=4 \text {. }
$$

This concluded the first optimum orthonormal matrix as

$$
\text { LSDC }-1=\begin{array}{lll}
4+j 5 & -2-j & 0 \\
2-j & 1-j & -6+j \\
0 & 6+j & -2-3 j \\
-2+j & -3+4 j & -6+j
\end{array} .
$$

It can be observed that the rational effects of $\sqrt{2}$ terms are completely removed from the orthogonal matrix realization. The SER probabilities corresponding to the LSDC-1 orthonormal matrix and the STBC are shown in Figure 4. Just for the sake of ease of computation the numbers of iterations are restricted to 100 for plotting the figure. It can be observed from Figure 4 that it needs great improvement in the performance of LSDC-1, as the SER probability for the LSDC-1 is even less than the existing STBC code validated earlier. It is because the orthonormal matrix in the LSDC-1 is having larger coefficients values for real and imaginary terms. Thus, it requires to rethink of optimizing the scalable coefficients.

\section{Case 2. LSDC-2}

For the sake of increasing the performance of the LSDC1 , let us start further optimizing the scalable coefficients. The coefficients are optimized by performing the various elementary matrix operations in the rows and columns of equation (10). The LSDC becomes 


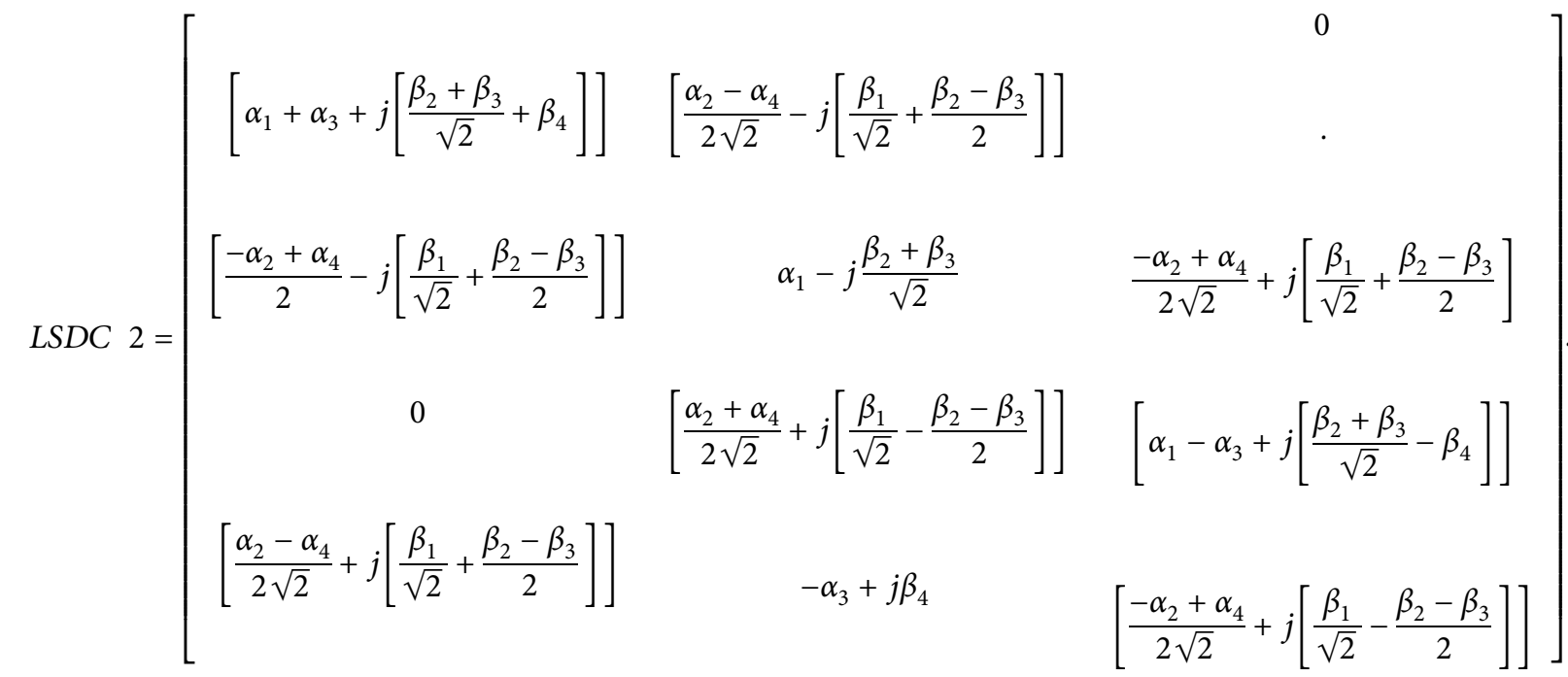

The values of $\alpha_{2}$ are reduced from $2 \sqrt{2}$ to $-\sqrt{2}$, and $\alpha_{4}$ are minimized to $\sqrt{2}$ from $4 \sqrt{2}$, respectively, in equation (14) of LSDC-1. The coefficient values for the LSDC-2 matrix are $\alpha_{1}=1 \alpha_{2}=-\sqrt{2} \alpha_{3}=3 \alpha_{4}=\sqrt{2}, \beta_{1}=\sqrt{2} \beta_{2}=1 / \sqrt{2} \beta_{3}=$ $1 / \sqrt{2} \beta_{4}=4$.

The LSDC-2 code vector is derived as

$$
L S D C-2=\begin{array}{lll}
4+j 5 & -j & 0 \\
-j & 1-j & j \\
0 & j & -2-3 j \\
-2+j & -3+4 j & j
\end{array} .
$$

The error probabilities corresponding to the LSDC-1 and LSDC-2 orthonormal matrix-based STBC are shown in Figure 5. It can be observed that good improvement in the SER performance is reported by the suggested modifications by LSDC- 2 compared to the LSDC-1. But still, the error rate is higher; thus it requires rethinking about optimizing the scalable coefficients.

\section{Case 3. LSDC-3}

To design the optimum LSDC-3, coefficients are further optimized and the values of $\alpha_{3}$ are reduced from 3 to 1 , and $\beta_{4}$ are minimized to 1 from 4 , respectively, in equation (16). Finally set the optimum values of the parameters to ensure that scalability is minimized by setting the coefficients as $\alpha_{1}=1, \alpha_{2}=-\sqrt{2}, \alpha_{3}=1, \alpha_{4}=\sqrt{2}, \beta_{1}=\sqrt{2}, \beta_{2}=1 / \sqrt{2}, \beta_{3}=$ $1 / \sqrt{2}$, and $\beta_{4}=1$.

Based on the experiences the orthonormal LSDC-3 matrix is given as

$$
L S D C-3=\begin{array}{lll}
2 & -j & 0 \\
-j & 1-2 j & j \\
0 & j & 0 \\
-2+j & -1-2 j & j
\end{array} .
$$

Case 4. LSDC-4
Based on the experience $\alpha_{2}$ and $\alpha_{3}$ are changed in the LSDC- 3 mentioned above; the coefficient values of LSDC-4 are, respectively, taken as $\alpha_{1}=1, \alpha_{2}=\sqrt{2}, \alpha_{3}=1, \alpha_{4}=\sqrt{2}$, $2 \beta_{1}=\sqrt{2}, \beta_{2}=1 / \sqrt{2}, \beta_{3}=1 / \sqrt{2}$, and $\beta_{4}=1$.

The respective orthonormal complex random matrix is reduced to

$$
\text { LSDC }-4=\begin{array}{lll}
2+2 j & -j & 0 \\
-j & 1-j & j \\
0 & 2 & 0 \\
j & -1+j & j
\end{array} .
$$

Case 5. O-LSDC

Now based on the experimental studies it is observed that reducing the complex coefficients can improve the performance of the BER of the system. The modified LSDC matrix O-LSDC is proposed which is obtained by the different types of matrix operations like elementary matrix operations, unitary matrix normalization technique, and coefficient mapping strategy. The obtained orthonormal O-LSDC matrix should satisfy the three constraints of the basic LSDC given in [1]. Figure 6 shows the flowchart for the proposed MIMO-O-LSDC system with the different higherorder PSK modulation techniques.

The O-LSDC can be obtained by following steps:

(i) Step 1: firstly, initialize the MIMO and LSD parameters $\mathrm{Nt}, \mathrm{Nr}$, Nitr count, and PSK order $\left(\alpha^{\prime} \mathrm{s}, \beta^{\prime}\right.$, $T, N_{\mathrm{t}}$ )

(ii) Step 2: initialize the popularly used Alamouti OSTBC matrix

(iii) Step 3: compare real and imaginary coefficients of $4 \times 3$ LSDC matrix and OSTBC

(iv) Step 4: mathematically formulate the linear equation of $\alpha^{\prime}$ s, $\beta^{\prime}$ s to get the optimal coefficient values

(v) Step 5: optimally search for the optimal LSDC parameters by solving the linear matrix equations and performing different matrix operations 


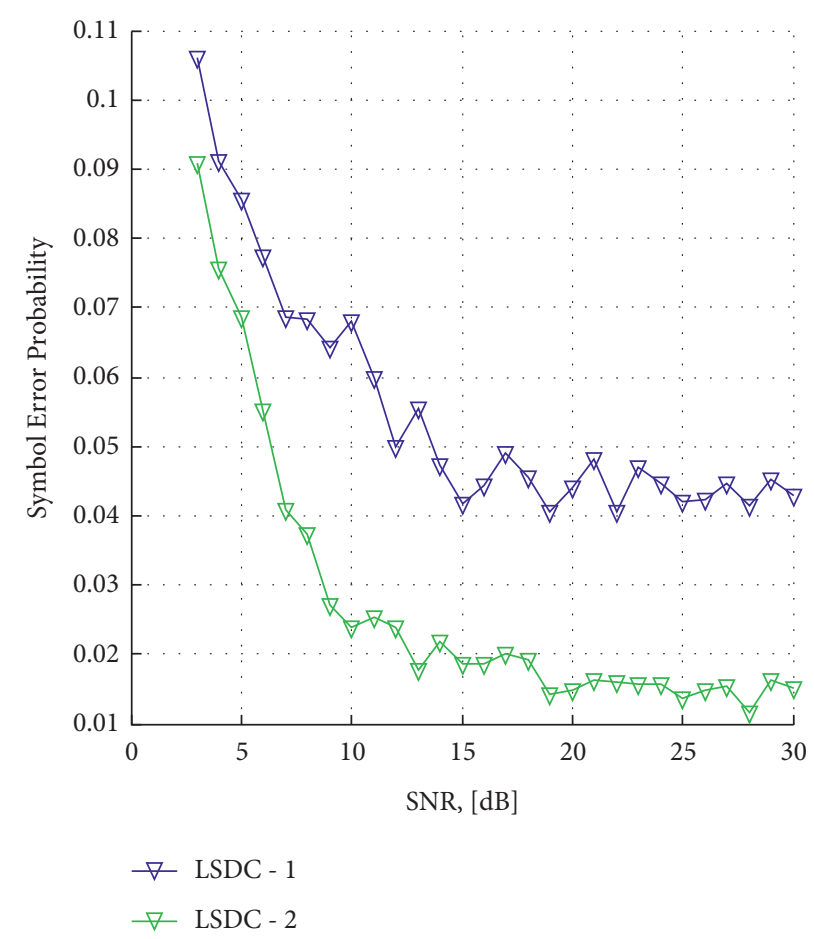

Figure 5: Comparison of SER for the LSDC-1 and the LSDC-2 performance.

(vi) Step 6: obtain the modified O-LSDC orthogonal matrix for the given set of antennas

The optimum LSDC matrix is obtained as

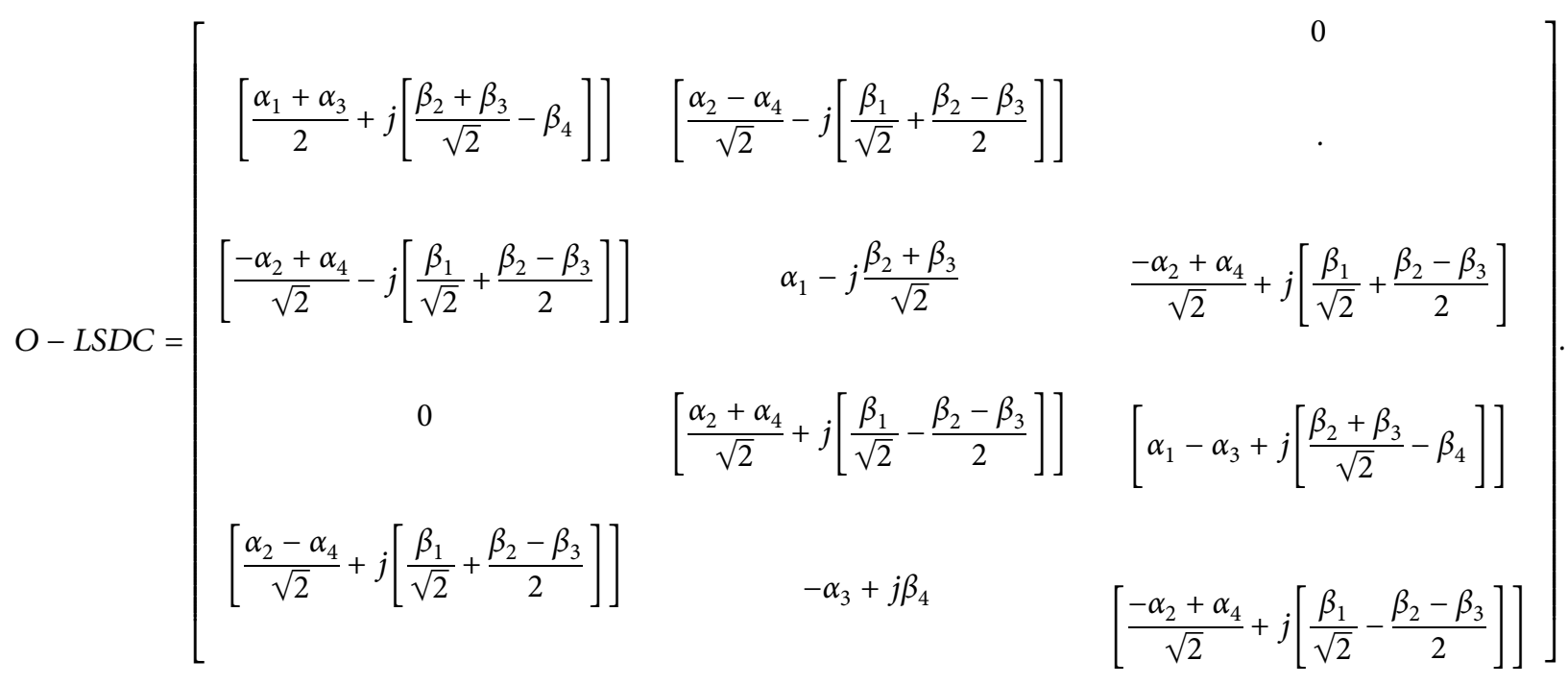

Corresponding to the coefficient used, the LSDC-4, the optimum O-LSDC orthonormal matrix, reduced to

$$
O-\text { LSDC }=\begin{array}{lll}
1 & -j & 0 \\
-j & 1-j & j \\
0 & 2 & 0 \\
j & -1+j & j
\end{array} .
$$

As the maximum real and imaginary parts are minimized to real numbers thus it is expected to improve the BER performance significantly.

\section{Simulation Results}

The results of the five models of LSDC are designed experimentally for enhancing the BER and SER of the LSDC 


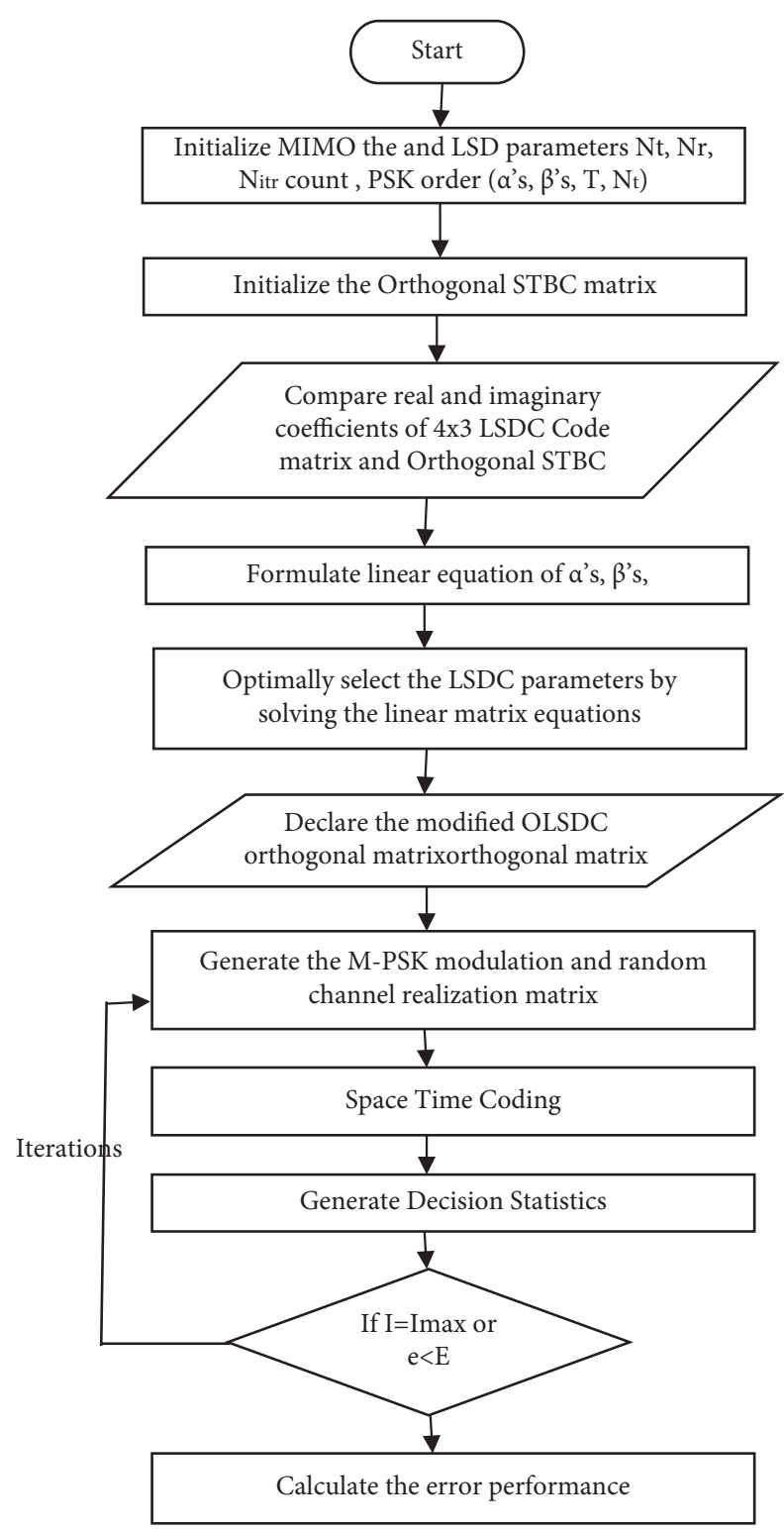

FIGURE 6: Flowchart for the proposed MIMO-O-LSDC system with the different higher-order PSK modulation techniques.

are presented in this section. Paper simulates the system for three antennas using the Rayleigh fading multiple-antenna communication system models. The five different orthonormal LSDC are used for performance evaluation. A hypothetical number of experiments are performed to design the optimum ST code vectors. The scale of the ST code vectors is varied based on the experiences and using linear equation solver for improving the performance of the BER of the $5 \mathrm{G}$ communication system. The work simulation and validation are done using MATLAB 2019a.

5.1. Evaluation of Error Probabilities. Figure 7 presents that the proposed O-LSDC code significantly outperforms the basic STBC code; clear improvement in SER is observed. It is clear from the results of Figure 7 that the significant improvement in error probabilities is achieved by the O-LSDC codes over the basic STBC codes. The proposed optimum O-LSDC outperforms the other mentioned codes. Another experiment is performed for the comparison of the BER for five various proposed LSDC codes with the STBC [9] for 1000 rounds of Monte Carlo simulation using the NRD distribution.

5.2. Impact of Monte Carlo Simulation. Evaluating the impact of the Monte Carlo simulation [24] over BER performance the number of iterations is varied and the BER and SER are plotted sequentially for the MIMO system as shown in Figures 8 and 9. As an experiment number of Monte Carlo iterations is from 1000 to 100000 and the performance is compared for the BER and proposed O-LSDC code for multiple-antenna system designs with NRD distribution. It is found that improvement can be achieved in BER with the increasing number of Monte Carlo iterations. 


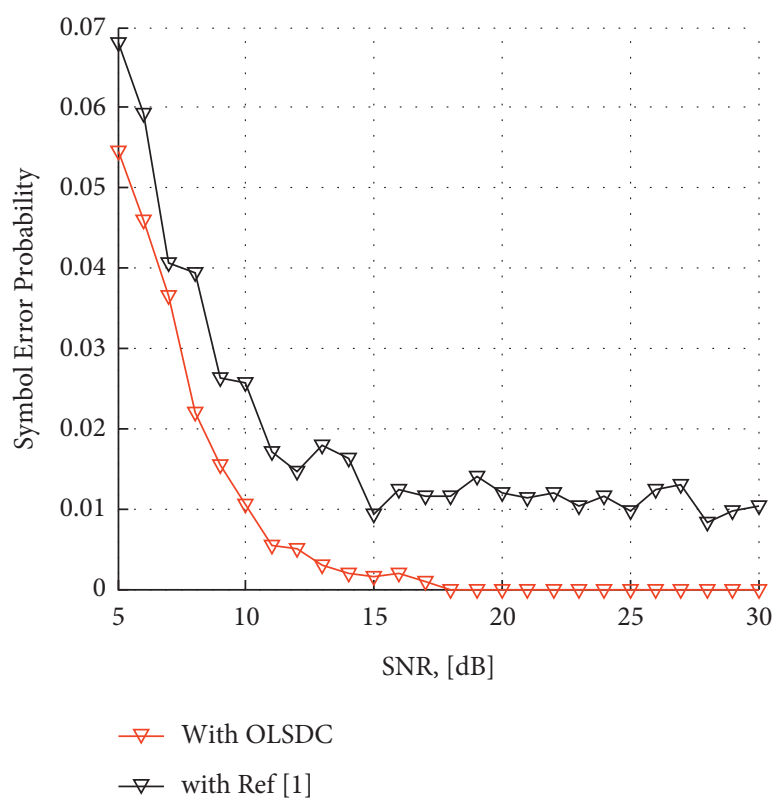

(a)

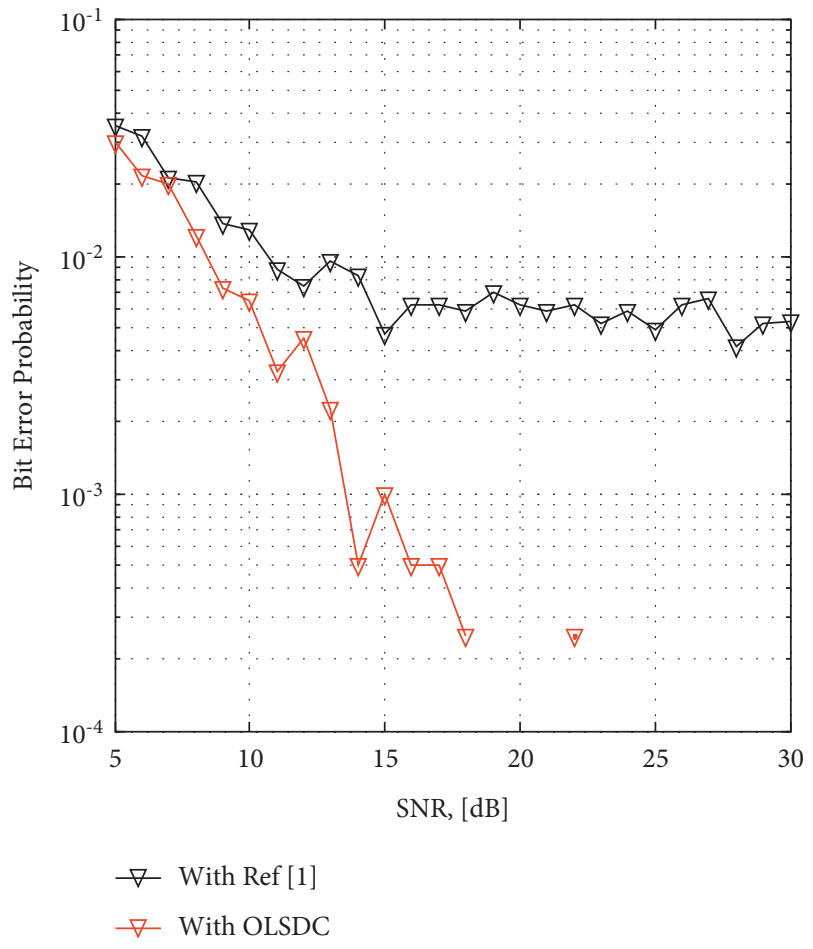

(b)

Figure 7: Performance comparison of the proposed O-LSDC code with [9]; (a) plot of the Symbol Error Probability (SEP) vs. SNR (in dB), (b) plot of the BER vs. SNR (in $\mathrm{dB}$ ).

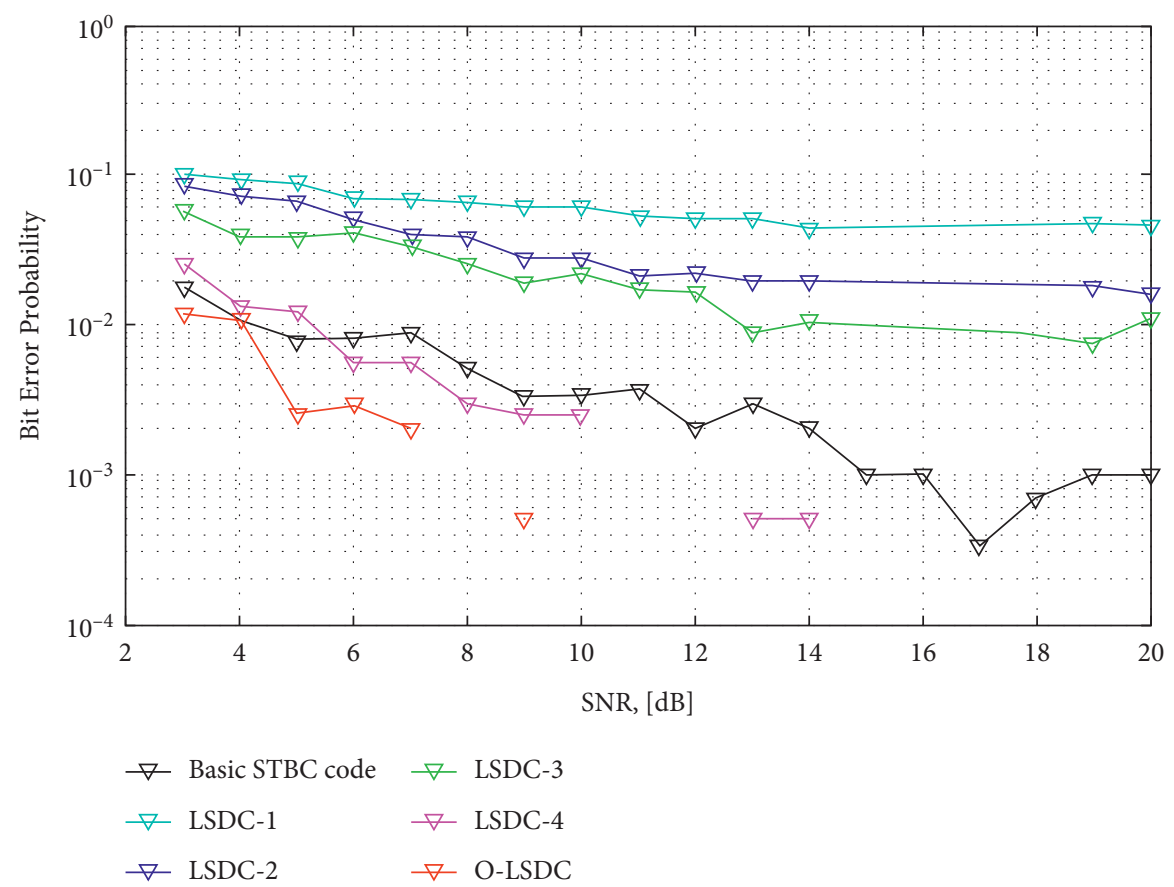

FIGURE 8: BER comparison for the various proposed LSDC codes for 1000 rounds of Monte Carlo simulation for NRD distribution.

5.3. Results Evaluation for O-LSDC for M-PSK. The performance is proposed to evaluate the M-PSK modulation by generating a random matrix of symbols. In this section, the results of the proposed multiple-antenna system over the different orders of the M-PSK modulations are evaluated. In M-PSK, modulation of the carrier signal takes on one of $M$ possible phase values depending on $(2 \pi(i-1) / M)$, where $i=1,2, \ldots, M$. The general 


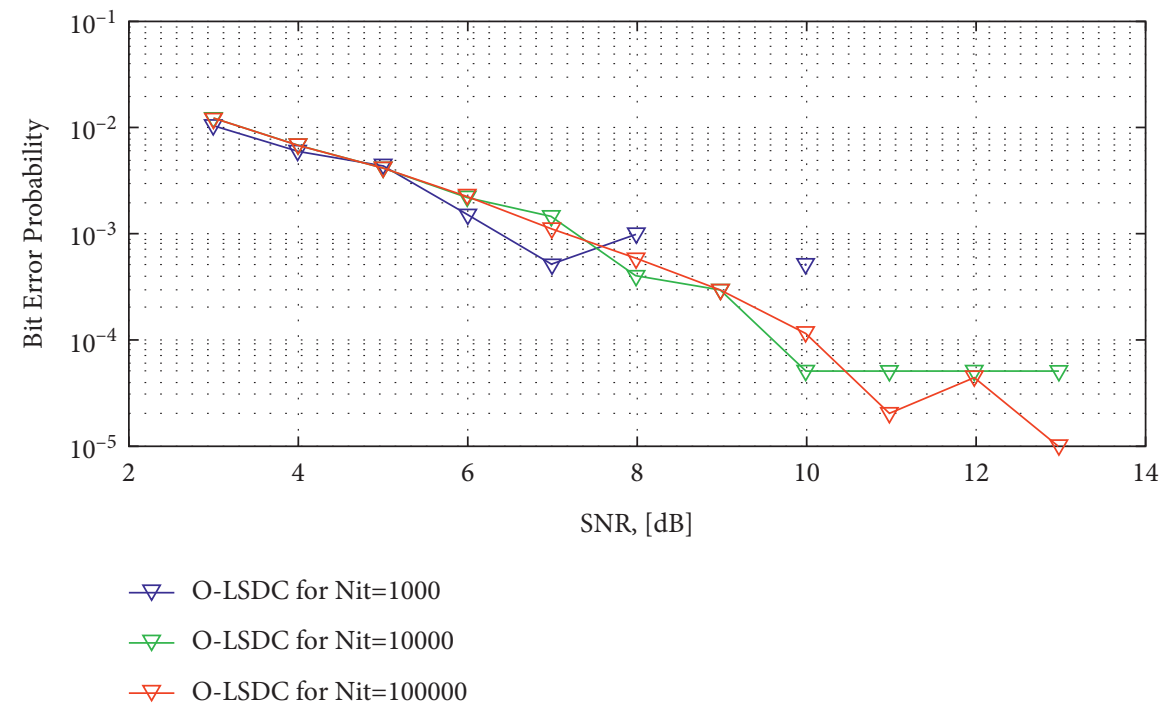

Figure 9: Comparison of the BER performance for proposed O-LSDC code with different number of Monte Carlo iterations from 100 to 100000 for NRD distribution.

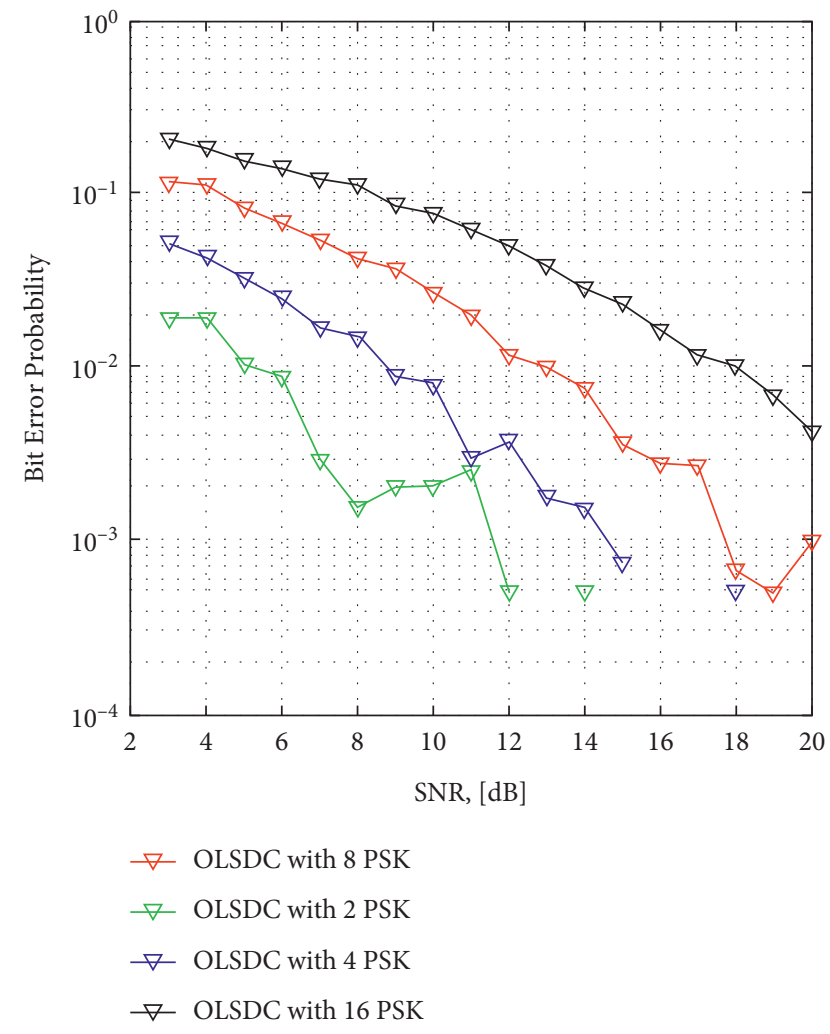

FIGURE 10: Comparison of the BER performance for the different modulation techniques for the fixed set of the Monte Carlo iterations of 1000 .

symbolical representation of the M-PSK modulation is given as

$$
V_{M P S K}^{i}(t)=\sqrt{\frac{2 E_{b}}{T_{b}}} \cos \left(2 \pi f_{c} t-\frac{2 \pi(i-1)}{M}\right) .
$$

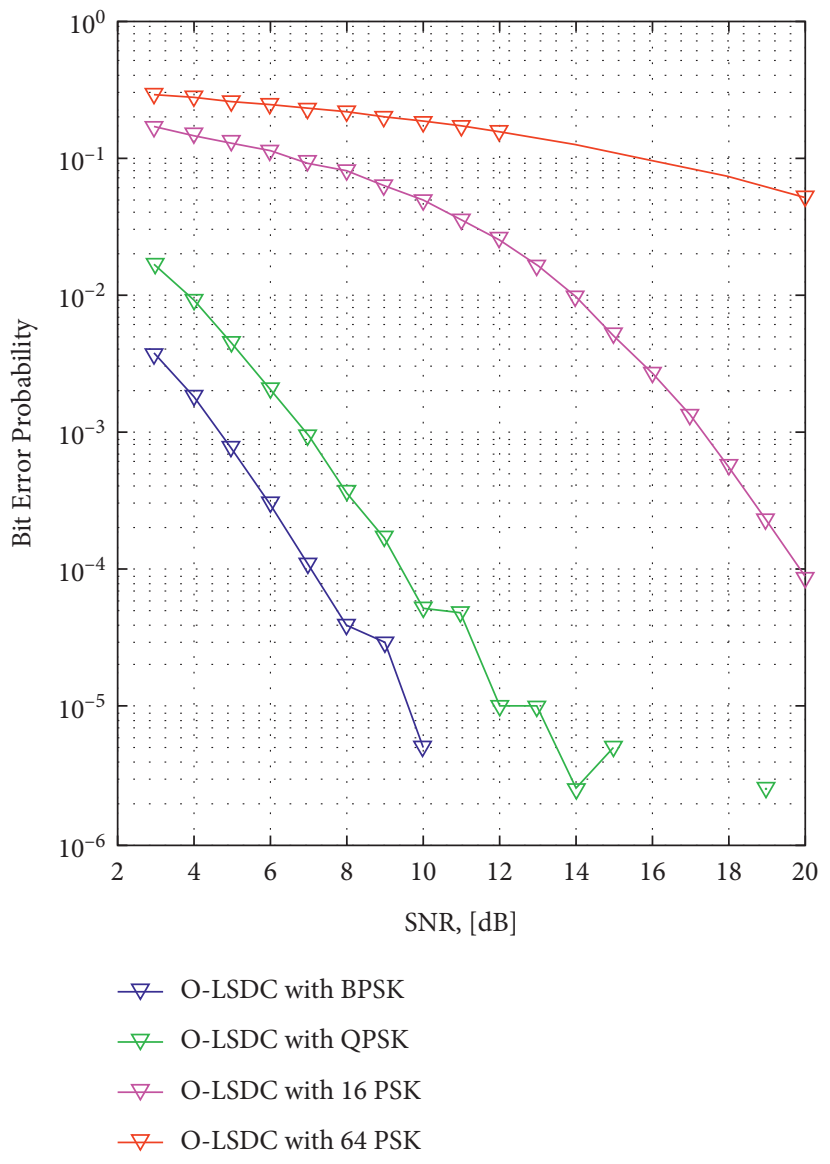

FIgURE 11: Evaluation of BER vs. SNR for the MIMO-O-LSDC system with the different higher-order PSK modulation techniques.

It is clear from the equation that increasing the order increases the number of phases, thus reducing the kind of orthogonality, and therefore it tends to increase the 
probability of the error. Figure 10 presents the proposed results comparison of BER for the different order of the PSK modulation techniques for the fixed set of the Monte Carlo iterations of 1000. It can be observed that the proposed O-LSDC improves the BER pattern and the BER continuously degrades with the increase in the SNR value of the system. The O-LSDC with 16 PSK even outperforms validated STBC code with the BPSK performance. The results are evaluated for the $3 / 4$ rate code for the 3 transmitting antenna systems. A good improvement is expected with the increasing number of iterations.

5.4. Performance under Massive PSK. Another experiment is performed for representing the efficiency of the proposed work where the performance is evaluated under the massive PSK scenario. The term massive represents the huge number of modulation phases. The order of the modulation for M-PSK is changed as the $m=2^{2 i}$ and set as [2, 4, 16, and 64] for the performance evaluation of BER vs. SNR in the multiple-antenna systems using O-LSDC as shown in Figure 11.

\section{Conclusions}

This work proposed a novel design of the LSDC that performs well in terms of BER and SNR in the multiple-antenna system for the $5 \mathrm{G}$ networks. In this work to find the optimum solution of the LSDC, five cases of O-LSDC are defined and then performance is evaluated against the BER vs. SNR in $\mathrm{dB}$. The scale of the ST code vectors is varied based on the different elementary matrix operations and using linear equation solver for improving the performance of the BER of the system. It can be observed that good improvement in the SER performance is reported by the suggested modifications in LSDC-2 compared to the LSDC-1. But still, the error rate is higher; thus it requires rethinking about optimizing the scalable coefficients. The proposed O-LSDC matrix is obtained by the unitary matrix normalization technique, which involves the different types of matrix operations like elementary matrix operations, matrix rotations, and matrix normalization and the obtained scalable matrix is much closer to the reparability property also. It is observed in the experimental studies that reducing the complex coefficients can enhance the BER performance of the system. Experimental results show that a significant improvement in error probabilities is achieved by the O-LSDC codes over the Alamouti STBC codes. It can be observed that the proposed MIMO-O-LSDC code improves the BER pattern and the BER continuously degrades with the increase in the SNR value of the system for the different M-PSK modulation, thereby increasing the performance of the $5 \mathrm{G}$ communication system. Future work in this topic includes the realization of the O-LSDC for the massive MIMO system, which is the key technology for the future wireless communication system and IoT connectivity.

\section{Data Availability}

The dataset used in this research work is available from the corresponding author.

\section{Conflicts of Interest}

The authors declare that they have no conflicts of interest.

\section{Authors' Contributions}

Conceptualization was done by by P. P., R. S., and P. S.; methodology, software validation, writing an original draft, review, editing, investigation, and project administration were done by by P. P; checking, review, and supervision were done by R. S. and P. S. All authors have read and agreed to the published version of the manuscript.

\section{Acknowledgments}

The authors are immensely grateful to the director of the Research Centre UIT, RGPV, Bhopal, India, and the honorable Vice-Chancellor of the RGPV, Bhopal, India, for their inspiration and motivation in the successful completion of the work.

\section{References}

[1] B. Hassibi and B. M. Hochwald, "High-rate codes that are linear in space and time," IEEE Transactions on Information Theory, vol. 48, no. 7, pp. 1804-1824, 2002.

[2] X. Xiaodong Wang, V. Krishnamurthy, and J. Jibing Wang, "Stochastic gradient algorithms for design of minimum errorrate linear dispersion codes in MIMO wireless systems," IEEE Transactions on Signal Processing, vol. 54, no. 4, pp. 1242$1255,2006$.

[3] N. Nan Wu and H. Gharavi, "Asynchronous cooperative MIMO systems using a linear dispersion structure," IEEE Transactions on Vehicular Technology, vol. 59, no. 2, pp. 779-787, 2010.

[4] J. Wu and S. D. Blostein, "High-rate diversity across time and frequency using linear dispersion," IEEE Transactions on Communications, vol. 56, no. 9, pp. 1469-1477, 2008.

[5] R. W. Heath Jr and A. J. Paulraj, "Linear dispersion codes for MIMO systems based on frame theory," IEEE Transactions on Signal Processing, vol. 50, no. 10, pp. 2429-2441, 2002.

[6] R. H. Gohary and T. N. Davidson, "Design of linear dispersion codes: asymptotic guidelines and their implementation," IEEE Transactions on Wireless Communications, vol. 4, no. 6, pp. 2892-2906, 2005.

[7] J. Wu, P. Xiao, M. Sellathurai, and S. D. Blostein, Linear Dispersion Codes for Wireless Communications, Nova Science Publishers, Hauppauge, NY, USA, 2008, https://www. researchgate.net/publication/270893757.

[8] M. Ming Jiang and L. Hanzo, "Unitary linear dispersion code design and optimization for MIMO communication systems," IEEE Signal Processing Letters, vol. 17, no. 5, pp. 497-500, 2010.

[9] V. Tarokh, N. Seshadri, and A. R. Calderbank, "Space-time codes for high data rate wireless communication: 
performance criterion and code construction," IEEE Transactions on Information Theory, vol. 44, no. 2, pp. 744-765, 1998.

[10] V. Tarokh, H. Jafarkhani, and A. R. Calderbank, "Space-time block codes from orthogonal designs," IEEE Transactions on Information Theory, vol. 45, no. 5, pp. 1456-1467, 1999.

[11] J.. Hamid, Space-Time Coding, Theory and Practice", Cambridge University Press, Cambridge, UK, 2005.

[12] J. Jibing Wang, X. Xiaodong Wang, and M. Madihian, "On the optimum design of space-time linear-dispersion codes," IEEE Transactions on Wireless Communications, vol. 4, no. 6, pp. 2928-2938, 2005.

[13] W. Jinsong and D. Steven, "Rectangular information lossless linear dispersion codes," IEEE Transactions on Wireless Communications, vol. 9, 2010.

[14] M. Nicola, C. Ernestina, and P. Ramjee, "Space-frequency linear dispersion codes for single carrier-frequency domain equalization," IEEE Transactions on Wireless Communications, vol. 8, pp. 5388-5393, 2009.

[15] G. Mabruk, W. Zhiyuan, and R. S. Yousef, "Design of an adaptive mimo system using linear dispersion code," IEEE Transactions on Wireless Communications, vol. 7, pp. 4739-4747, 2008.

[16] W. Jinsong and D. Steven, "High-rate diversity across time and frequency using linear dispersion," IEEE Transactions on Communications, vol. 56, 2008.

[17] W. Wang, F.-C. Zheng, A. Burr, and M. Fitch, "Design of delay-tolerant linear dispersion codes," IEEE Transactions on Communications, vol. 60, no. 9, pp. 2560-2570, 2012.

[18] H. X. Nguyen, H. H. Nguyen, and T. Le-Ngoc, "Optimization of linear dispersion codes for wireless relay networks," IEEE Signal Processing Letters, vol. 16, no. 5, pp. 366-369, 2009.

[19] E. Biglieri, Y. Hong, and E. Viterbo, "On fast-decodable spacetime block codes," IEEE Transactions on Information Theory, vol. 55, no. 2, pp. 524-530, 2009.

[20] X. Dai, S. Cheung, and T. Yuk, "A fast-decodable code structure for linear dispersion codes," IEEE Transactions on Wireless Communications, vol. 8, no. 9, pp. 4417-4420, 2009.

[21] X. Mestre and D. Gregoratti, "Diversity analysis of randomized linear dispersion codes in a half-duplex amplify-andforward multiple-relay system," IEEE Transactions on Information Theory, vol. 59, no. 5, pp. 2936-2959, 2013.

[22] S. Kopparty and S. Saraf, "Local testing and decoding of highrate error correcting codes. Proc. Electron. Colloq. Computing Complex," ECCC), vol. 47, pp. 46-66, 2017.

[23] C. Tian, H. Sun, and J. Chen, "Capacity-achieving private information retrieval codes with optimal message size and upload cost," IEEE Transactions on Information Theory, vol. 65, no. 11, pp. 7613-7627, 2019.

[24] R. Y. Rubinstein and D. P. Kroese, Simulation and the Monte Carlo Method, John Wiley \& Sons, Hoboken, NJ, USA, 2017, http://www.ru.ac.bd/wp-content/uploads/ sites/25/2019/03/308_03_Rubinstein_Simulation-and-theMonte-Carlo-Method-Wiley-2017.pdf. 